\title{
Gridless Ion Acceleration Systems for Time-of-Flight Mass Spectrometry*
}

\author{
Lothar Schmidt, Hartmut Jungclas, Hans-Walter Fritsch, and Peter Köhl \\ Klinische Nuklearmedizin, Philipps-Universität Marburg, Marburg, Germany
}

Pointlike ion sources allow the application of gridless acceleration systems in time-of-flight mass spectrometry (TOF/MS). When ions are extracted from large sample areas according to the applied ionization method and sample genmetry, the application of electrostatic lenses for acceleration seems to be difficult. Inhomogeneous extraction fields are likely to induce acceleration time variations for ions emerging from different locations on the sample. We investigated gridless acceleration systems with the help of computer simulations. An appropriate solution for TOF/MS was found and experimentally tested, combining the features of compactness and a wide-acceptance aperture with simple principles of construction. (J Am Soc Mass Spectrom 1993, 4, 782-786)

$\mathrm{T}$ he history of mass spectrometry is closely connected with the development of ion sources and ion optics. High-resolution sector-field instruments required the application of lens systems to transfer the ions from the source to narrow entrance apertures and finally to focus them precisely at the exit slit. In contrast to such instruments, time-of-flight (TOF) mass spectrometers can work without any sophisticated ion optical element. High resolution has been achieved by the use of simple, homogeneous accelerating fields in ion sources and ion mirrors $[1,2]$.

More recently, focusing elements in TOF systems have been investigated and installed, such as the Einzel lens [3], toroidal sector field [4,5] and gridless ion mirror [6]. The acceleration of ions by a lens system has also become a standard technique for TOF mass spectrometers using point-like ion sources. With such lenses it is easy to produce a parallel beam of accelerated ions; however, when the ions are extracted from large sample areas [up to $1 \mathrm{~cm}^{2}$ in $\left({ }^{252} \mathrm{Cf}-\right)$ plasma desorption mass spectrometry (PDMS)], the application of electrostatic acceleration lenses seems to be disadvantageous because inhomogeneous electrostatic fields will lead to varying acceleration times for ions of different sites of origin. This of course endangers the mass resolution of a TOF mass spectrometer.

Nevertheless, the use of gridless acceleration systems offers the chance to enhance ion transmission and to reduce beam divergence. A first experimental approach [7] and our own experience with gridless acceleration in PDMS encouraged us to search for an optimized solution by computer simulations. The

*Dedicated to Ronald D. Macfarlane on the accasion of his 60th birthday.

Address reprint requests to Hartmut Jungclas, Klinische Nuklearmedizin, Philipps-Ĺniversität Marburg, D-35033 Marburg, Germany. optimizations aimed for a large area of acceptance, extreme compactness, and simplicity.

\section{Methods}

Simulations of electrostatic lenses were performed on a 33-MHz PC-AT with the PC version of the SIMION code [8]. The trajectories were calculated for ions $(m / z$ 100) desorbed from a $7-\mathrm{mm}$ diameter target $(8-\mathrm{kV}$ acceleration voltage) with $0-\mathrm{eV}$ initial energy. Equidistant TOF markers $(0.2 \mu \mathrm{s})$ revealed the development of the ion front curvature in time along the beam (SIMION parameters: grid spacing $0.25 \mathrm{~mm}, 400 \times 40$ points mirrored, voltage scaling $1 \mathrm{~V}$, accuracy level 100 , cylindrical symmetry).

Two calculated lens versions were constructed and experimentally tested in comparison with conventional acceleration (90\% transmission grid, sample grid distance $8.5 \mathrm{~mm}$ ). The measurements were performed on a noncommercial linear TOF PDMS instrument that is mainly used for quantitative measurements of drug concentrations in blood serum and for the analysis of peptides. The samples were distributed on a surface of $0.4 \mathrm{~cm}^{2}$ (radius $3.5 \mathrm{~mm}$ ) and irradiated by about 400 fission fragments per second emerging from a $3-\mu \mathrm{Ci}$ ${ }^{252} \mathrm{Cf}$ source. The desorbed and accelerated ions drift in a field-free tube and finally directly strike the stop detector face (microchannel plates, active surface diameter $32 \mathrm{~mm}$ ) at a distance of $73 \mathrm{~cm}$ from the ion source.

TOF mass spectra (1-ns resolution) were acquired from samples of verapamil hydrochloride prepared from a $1-\mathrm{mg} / \mathrm{mL}$ solution in methanol. The molecular ion distribution at approximately $m / z 455$ was used to compare mass resolution and ion yield obtained with the different acceleration systems. Intensity losses due 
to an eventually increased beam divergence are most likely indicated by a decreased yield of protons $[9,10]$.

\section{Results of Computer Simulations}

The basic scheme of the type of acceleration lens developed here is shown in Figure 1. Two annular electrodes with aperture radius $r$ face the sample electrode at distances $d_{0}$ and $D$. The middle electrode with thickness $d$ is at potential $U_{1}$ and may be called the extraction, or focusing, electrode. The main acceleration electrode is assumed to be at ground potential according to the field-free drift region. The acceleration voltage $U_{0}>U_{1}$ is applied to the sample electrode. Nearly homogeneous electrostatic fields $E_{0}$ and $E_{1}$ are generated in the gaps between the electrodes at radial distances exceeding $r$. In the case where $U_{1}$ is adjusted to a value fulfilling the condition $E_{1}>E_{0}$, this electrode configuration corresponds to the scheme of a simple immersion lens.

The simulation of many different gridless acceleration lenses designed according to the above-described scheme revealed an essential feature of this compact and simple design: The potential, thickness, and position of the semicircular-shaped ring electrode determine the form of the extraction field and thus the propagation trajectories of the emerging ions. Its potential $U_{1}$ is adjusted to yield parallel rays. Its thickness $d$ determines the acceleration time as a function of the radial distance of the trajectories. Its position $d_{0}$ influences the imaging properties (lens aberrations) and thus the acceptance radius.

SIMION calculates the distances that the ions have drifted after selected time intervals. By controlling the ion positions at these flight times, it is possible to find the trajectories that potentially contribute to peak broadening. Figures 2 and 3 demonstrate the influence of thickness $d$ on the ion front curvature $\left(U_{1}\right.$ is adjusted to yield a parallel beam). Obviously, the curva-

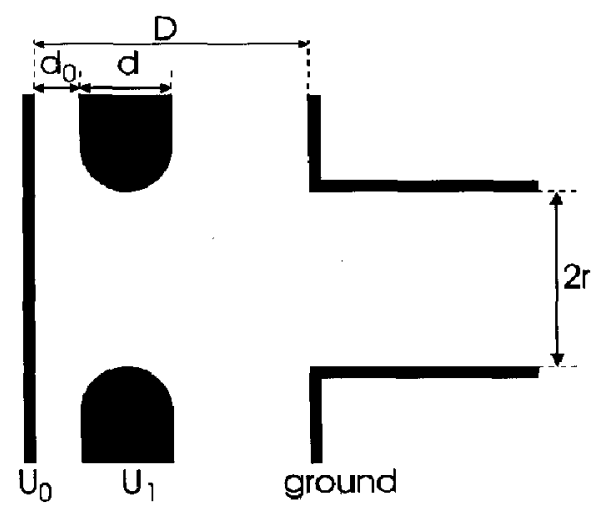

Figure 1. Geometric parameters and potentials of the acceleration lens. ture is inverted when the thickness becomes too large. It is concluded that for any set of the geometric lens parameters $d_{0}, D$, and $r$, there is an optimum thickness $d=d_{\text {opt }}$, resulting in parallel rays and good timing behavior. Moreover, it was found that for a symmetric solution defined by the condition $r=d_{0}+d / 2$, the lens aberrations are minimized, leading to a high acceptance radius. When this symmetry condition is fulfilled, the optimum thickness $d_{\mathrm{vpt}}$ can be calculated by

$$
d_{\mathrm{opt}}=\left(r+d_{0}\right) 2 r / D
$$

resulting in the optimum electrode position,

$$
d_{0}=r(D-r) /(D+r)
$$

The optimum potential $U_{1}$ of the ring electrode in a given geometry (characterized by $d_{0}, d, D$, and $r$ ) at a total acceleration potential $U_{0}$ is calculated to first order by

$$
U_{1} / U_{0}=1-d_{0} / 2 D-d / 4 D
$$

More detailed investigations resulted in a correction term that contains the $r$ dependence:

$$
\begin{aligned}
U_{1} / U_{0}= & \left(1-d_{0} / 2 D-d / 4 D\right) \\
& \cdot\left[1+d / 3 D-r / 3\left(D+d_{0}\right)\right]
\end{aligned}
$$

This formula is very precise for thickness $d=d_{\mathrm{ppt}}$ and is a good approximation for any $d$. Small variations of the form of the exit aperture lead to small deviations without basically changing the quality of the lens. For example, a slight increase in $U_{1}$ is necessary when the sharp inner edges are massively rounded off.

A very compact solution with large acceptance $(50 \%$ of open area) and small aberrations was found (Figure 4) at

$$
r=0.4 D, \quad d=0.45 D, \quad d_{0}=D / 6, \quad U_{1}=0.833 U_{0}
$$

It is emphasized that under these conditions, the acceleration lens is not point-to-point focusing the sample surface at the detector plane. The design is simply based on the principle that the focusing and defocusing fields have to compensate each other, yielding parallel trajectories in the field-free drift space and avoiding peak broadening caused by varying acceleration times. A focusing effect can, however, be achieved by a slight increase in the electrode potential $u_{1}$ and distance $d_{0}$. Extended investigations are necessary to find calculable solutions with well-defined finite focal distances. 


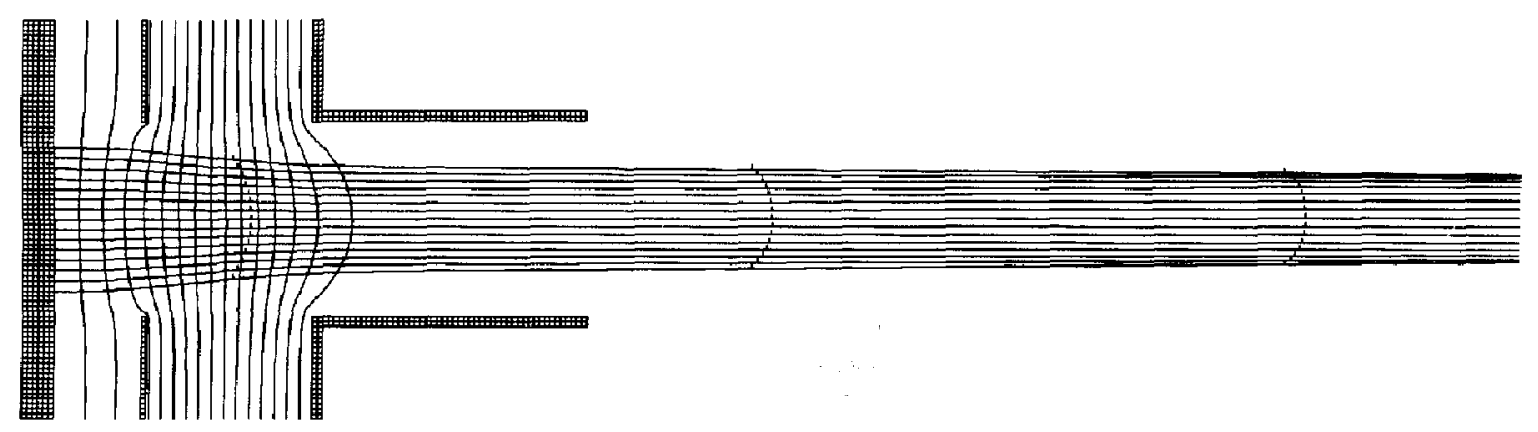

Figure 2. Beam propagation for electrode thickness $d<d_{\text {opt }}$ i equipotential contours plotted in steps of $500 \mathrm{~V}$ (acceleration voltage $8 \mathrm{kV}$ ).

\section{Experimental Results}

The result obtained with conventional grid acceleration was compared with the performance of an acceleration lens of similar dimensions to the optimized solution displayed in Figure 4 . A voltage of $8 \mathrm{kV}$ was applied to accelerate the inns desnrhed from the sample, which was prepared by drying a few microliters of a $1-\mathrm{mg} / \mathrm{mL}$ solution of verapamil hydrochloride in methanol on an aluminized foil. This antiarrhythmic drug was chosen to serve as a test substance because high molecular ion yields at $m / z 453$ and 455 allow fast and convenient visual control of the achieved mass resolution. Figure 5a shows the molecular ion region of the $\mathrm{PD}$ mass spectrum obtained by conventional acceleration by means of a $90 \%$ transmission grid at a distance of $8.5 \mathrm{~mm}$ from the sample surface.

The corresponding spectrum for the acceleration by the gridless system is shown in Figure 5b. Obviously, the mass resolution is decreased significantly. This effect is caused by the low acceleration field strength close to the sample surface. Here the ions are slow, and the velocity differences due to the initial energy distribution are high. Thus, ions of equal mass-tocharge ratios but different initial energies have time to drift apart. With increasing energies, the velocity dif- ferences are strongly decreasing, whereas the energy differences are conserved (velocity-bunching effect).

As a consequence, it is necessary to increase the acceleration field strength near the sample surface to accomplish higher mass resolutions with a gridless acceleration system. According to this requirement, a modified system was constructed (Figure 6). An additional electrode with the same dimensions as the focusing electrode was mounted in front of the exit aperture. In agreement with results of SIMION simulations, its potential was fixed at $U_{2}=-U_{0}$, and the focusing electrode was set at $U_{1}=2 / 3 U_{0}$. The aperture radius $r$ exceeded the radius of the active sample surface by only $15 \%$.

The mass resolution was now the same as that obtained with the homogeneous $8.5-\mathrm{mm}$ acceleration field (Figure 5c). The intensity of the molecular ions was about $10 \%$ higher, but this result could reflect the influence of slightly different preparation conditions. The proton intensity should be a much more reliable indicator of an enhanced ion transmission. Surprisingly, the gridless system exhibits a $30 \%$ higher proton intensity that cannot be totally explained by the absence of the grid; but it is known that protons are desorbed with very high divergencies by fission fragments $[9,10]$ and that a grid increases the beam diver-

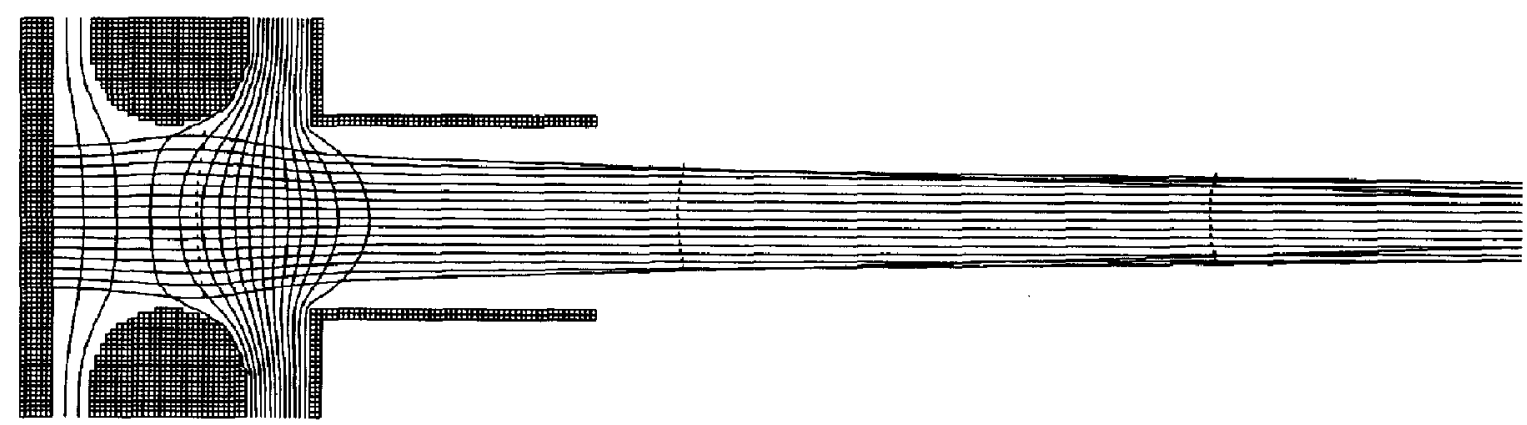

Figure 3. Beam propagation for electrode thickness $d>d_{\text {opt }}$ equipotential contours plotted in steps of $500 \mathrm{~V}$ (acceleration voltage $8 \mathrm{kV}$ ). 


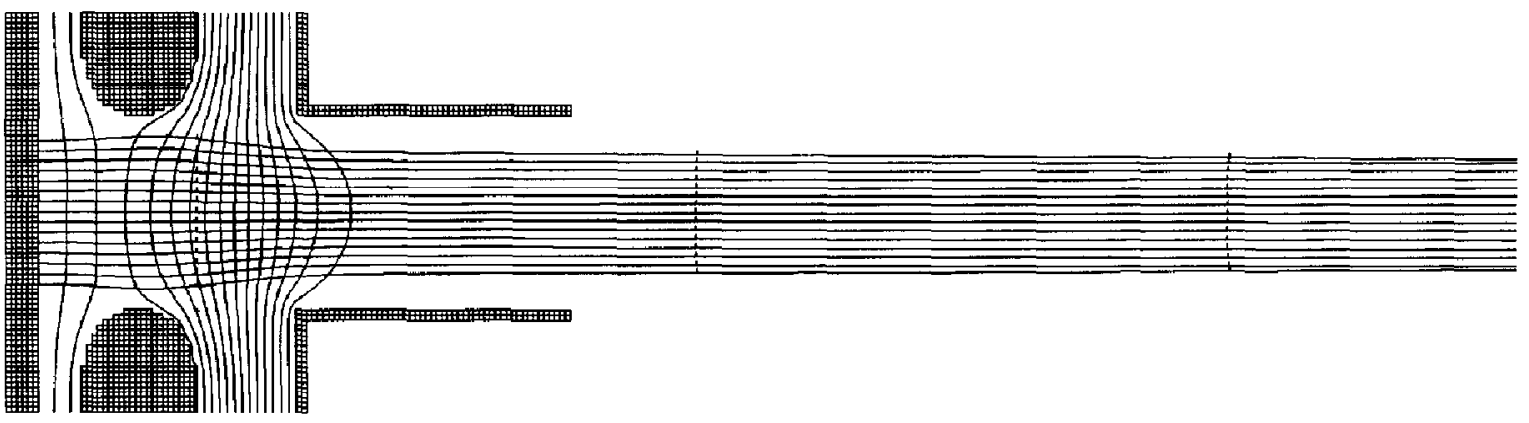

Figure 4. Beam propagation for electrade thickness optimized for $r=0.4 D, d_{0}=1 / 6 D, d=0.45 D$, $U_{1}=0.833 U_{n}$; equipotential contours plotted in steps of $500 \mathrm{~V}$ (acceleration voltage $8 \mathrm{kV}$ ).

gence. Thus, it can be concluded that the gridless system is able to reduce ion losses caused by high radial velocities in TOF systems with long flight distances and/or small detector diameters.

\section{Conclusions}

A simple electrostatic lens can be efficiently applied for ion acceleration in TOF mass spectrometry (TOF MS), especially in PDMS. The position, form, and thickness

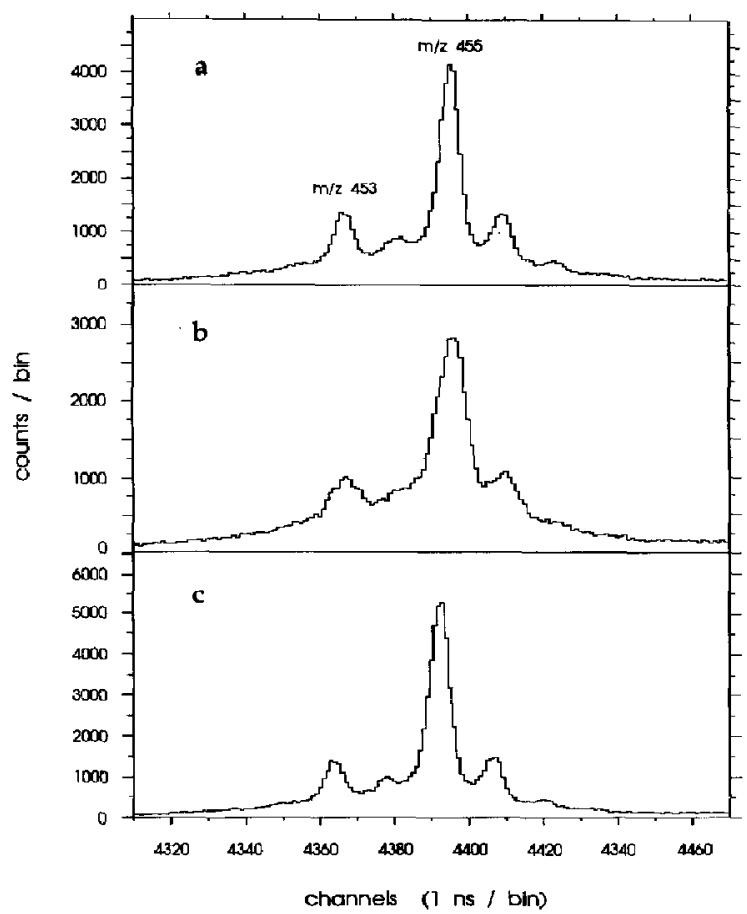

Figure 5. Molecular ions of verapamil hydrochloride employing (a) grid, (b) single-electrode lens, and (c) twin-electrode lens (see Figure 6) for acceleration. of a central ring electrode are essential for optimizing the ion extraction field. With the help of the formulas derived, it is possible to build up such an optimized acceleration system without the need for further computer simulations.

The first TOF MS experiments employing such an optimized lens yielded the expected performance: The ion yield increased owing to the absence of a grid and the reduced beam divergence, whereas the mass resolution decreased. The decrease in mass resolution is due to the weak ion extraction field. A field strength similar to a grid system was achieved with a modified acceleration lens. Mass resolution was then as high as that obtained with grid acceleration. The molecular ion yield increased by about $10 \%$, as expected; the proton intensity by $30 \%$. To enhance the mass resolution of the single ring electrode system, it is necessary to raise the acceleration voltage or to compensate for the ion energy distribution by means of a reflectron.

The results demonstrate the possibility of applying a gridless ion acceleration system in TOF mass spectrometers that makes use of extended ion sources. The solutions presented here are compact and can easily be constructed and installed. The need for additional high-voltage supplies might be reduced by the application of a voltage divider. The advantage of avoiding ion losses caused by a grid is obvious. In particular, for instruments with a long flight path, the reduction of beam divergence will significantly enhance ion transmission. In instruments with a shorter flight path, smaller detectors could be installed as a consequence of the focusing effect of the acceleration lens.

The combination of gridless acceleration with a gridless reflectron is very promising because in this case, the disadvantage of the weak acceleration field in the vicinity of the sample surface is no longer a limiting factor for mass resolution. In addition, gridless acceleration offers the possibility of shooting ionizing laser or ion beams through the open aperture of the system onto the target surface.

The gridless ion acceleration systems described in this report are not the only possible solutions, but they 


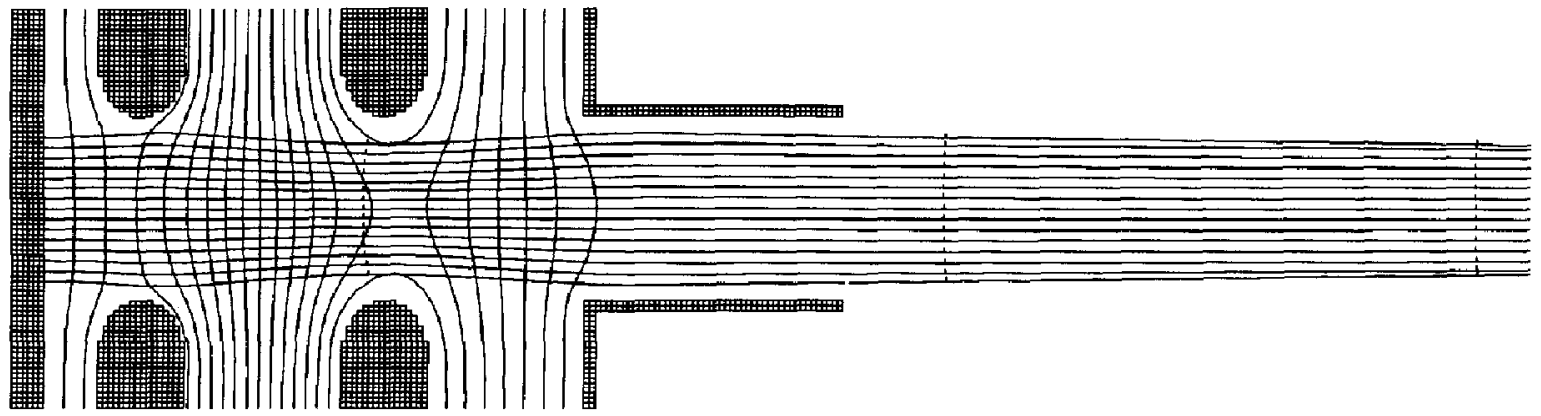

Figure 6. Acceleration system with twin electrodes (middle electrode potentials: $U_{1}=2 / 3 U_{0}$. $U_{2}=-U_{0}$; geometry: $\left.d_{0}=0.1 \mathrm{D}, d_{1}=0.55 \mathrm{D}, r=0.18 \mathrm{D}, d=0.16 \mathrm{D}\right)$ yielding higher extraction field strength than single-electrode lens; equipotential contours plotted in steps of $1 \mathrm{kV}$ (acceleration voltage $8 \mathrm{kV}$ ).

are optimized with regard to simplicity, compactness, and ion acceptance.

\section{Acknowledgment}

Financial support by Deutsche Forschungsgerneinschaft, Bonn, is thankfully acknowledged.

\section{References}

1. Wiley, W. C.; McLaren, I. M. Rev. Sci. Instrum. 1955, 26, 1150.

2. Mamyrin, B. A.; Karatev, V. I.; Shmikk, D. K.; Zagulin, V. A. Sov. Phys. JETP (Engl. Transl.) 1973, 37, 45.

3. Jonsson, G.; Hedin, A.; Håkansson, P.; Sundqvist, B. U. R.; Bennich, H.; Roepstorff, P. Rapid Commun. Mass Spectrom. 1989, 3, 190 .
4. Poschenrieder, W. R. Int. J. Mass Spectrom. Ion Phys. 1972, 9, 357.

5. Stcffens, P.; Niehuis, E.; Friese, T.; Greifendorf, D.; Benringhoven, A. I. Vac. Sct. Technol. 1985, A3, 1322.

6. Grix, R.; Kutscher, R.; Li, G.; Grüner, U.; Wollnik, H. Rapid Comm. Mass Spectrom. 1988, 2, 83.

7. Della Negra, S.; Deprun, C.; Le Beyee, Y.; Röllgen, F.; Standing, K.; Monard, B.; Bolbach, G. Int. I. Mass Spectrom. Ion Processes 1987, 75, 319

8. Dahl, D. A.; Delmore, J. E. The SIMION PC/PS2 User's Manual Version 4.0, EGG-CS-7233 Revision 2; Informal Report; Idaho Falls National Engineering Laboratory: Idaho Falls, ID, 1988.

9. Wolf, B.; Mudgett, P. D.; Macfarlane, R. D. I. Am. Soc. Mass Spectrom. 1990, 1, 28.

10. Moshammer, R.; Matthäus, R.; Wien, K.; Bulbach, G. In Ion Formation from Organic Solids (IFOS V); Hedirl, A,; Sundqvist, B. U. R.; Benninghoven, A., Eds.; Wiley: New York 1990; p 17 . 